\title{
Monte Carlo results for the hydrogen Hugoniot
}

\author{
V. Bezkrovniy, ${ }^{1}$ V. S. Filinov, ${ }^{2}$ D. Kremp,${ }^{3}$ M. Bonitz,${ }^{4}$ M. Schlanges,${ }^{1}$ \\ W. D. Kraeft, ${ }^{1}$ P. R. Levashov, ${ }^{2}$ and V. E. Fortov ${ }^{2}$ \\ ${ }^{1}$ Institut für Physik, Ernst-Moritz-Arndt-Universität Greifswald, \\ Domstrasse 10a, D-17487, Greifswald, Germany \\ ${ }^{2}$ Institut for High Energy Density, Russian Academy of Science, \\ Izhorskaja str. 13/19, 125412 Moscow, Russia \\ ${ }^{3}$ Fachbereich Physik, Universität Rostock, \\ Universitätsplatz 3, D-18051 Rostock, Germany \\ ${ }^{4}$ Christian-Albrechts-Universität zu Kiel, \\ Institut für Theoretische Physik und Astrophysik, \\ Leibnizstr. 15, 24098 Kiel, Germany
}

(Dated: October 29, 2018)

\begin{abstract}
We propose a theoretical Hugoniot obtained by combining results for the equation of state (EOS) from the Direct Path Integral Monte Carlo technique (DPIMC) and those from Reaction Ensemble Monte Carlo (REMC) simulations. The main idea of such proposal is based on the fact that DPMIC provides first-principle results for a wide range of densities and temperatures including the region of partially ionized plasmas. On the other hand, for lower temperatures where the formation of molecules becomes dominant, DPIMC simulations become cumbersome and inefficient. For this region it is possible to use accurate REMC simulations where bound states (molecules) are treated on the Born-Oppenheimer level using a binding potential calculated by Kolos and Wolniewicz. The remaining interaction is then reduced to the scattering between neutral particles which is reliably treated classically applying effective potentials. The resulting Hugoniot is located between the experimental values of Knudson et al. 1] and Collins et al. 2].
\end{abstract}

PACS numbers: 64.30.+t, 05.30.-d, 62.50.+p 
The H-plasma is a very important and interesting many particle system. Hydrogen is the simplest and at the same time the most abundant element in the universe. Due to its high relevance for modern astrophysics, inertial confinement fusion and fundamental understanding of condensed matter, hydrogen continues to be actively studied both, experimentally 1, 2, 3, 4, 5, 6] and theoretically [7, 8, 9, 10, 11, 12, 13, 14]. At high temperatures and pressures, the hydrogen behavior is defined by the interaction between free electrons and protons (plasma state). With decreasing temperature, the contribution of bound states such as atoms and molecules to the EOS of hydrogen becomes of increasing importance, which at low temperatures completely define the hydrogen properties. Furthermore, as pointed out in many papers (Norman and Starostin [15], Ebeling et al. 16], Haronska et al. [17], Saumon and Chabrier [18]) there are strong theoretical arguments for a phase transition between two plasma phases. This issue which is of importance, for example, for models of Jovian planets is still actively debated. Among other important questions we mention the high-pressure compressibility, details of the pressure ionization and dissociation.

For this reason, in the last decades considerable experimental and theoretical investigations were carried out to accurately determine the EOS of hydrogen at high pressures. Experimentally, the EOS for this region can be obtained using shock-wave techniques. The results of these experiments are usually discussed in form of an Hugoniot

$$
E=E_{0}+\frac{1}{2}\left(p+p_{0}\right)\left(\frac{1}{\rho}-\frac{1}{\rho_{0}}\right),
$$

where the specific internal energy $E$ at a state with the density $\rho$ and the pressure $p$ is connected to the initial conditions with the density $\rho_{0}$, the pressure $p_{0}$ and the internal energy $E_{0}$.

One of the well established experimental techniques for the creation of shock waves uses gas gun devices. With gas gun experiments, Nellis et al. [3] reached maximum pressures of 20 GPa and temperatures of $7000 \mathrm{~K}$. More advanced techniques, the laser-driven experiments used by Collins et al. 22] and Da Silva et al. 4], allow to reach pressures up to 300 GPa. At such pressures, as expected, hydrogen transforms from a molecular to a metallic state [5]. The results of laser-driven experiments have shown an unusual high compression $\rho / \rho_{0}=6$ of deuterium, which deviates significantly from a maximum compression of $\rho / \rho_{0}=$ 4.4 obtained within the SESAME EOS [19]. However, the experiments of Knudson et al. 1] which used magnetically driven flyer techniques (Z-pinch) do not support such high 
TABLE I: Thermodynamic properties of deuterium plasma calculated by DPIMC

\begin{tabular}{c|cc|cc|cc|c|c}
\hline \hline & \multicolumn{2}{|c|}{$r_{s}=1.7$} & \multicolumn{2}{|c|}{$r_{s}=1.86$} & \multicolumn{2}{|c|}{$r_{s}=2$} & \\
$T, \mathrm{~K}$ & $P, \mathrm{GPa}$ & $E, \mathrm{eV}$ & $P, \mathrm{GPa}$ & $E, \mathrm{eV}$ & $P, \mathrm{GPa}$ & $E, \mathrm{eV}$ & $\rho_{H}, \mathrm{~g} / \mathrm{cm}^{3}$ & $P_{H}, \mathrm{GPa}$ \\
\hline 15625 & 227.01 & -18.9953 & 101.41 & -9.6854 & & & 0.8539 & 111.32 \\
31250 & 186.25 & -9.94854 & & & 134.30 & -6.0186 & 0.8370 & 160.53 \\
62500 & & & 314.11 & -1.2281 & 261.05 & -0.1776 & 0.8104 & 306.69 \\
$1.25 \cdot 10^{5}$ & & 7.9579 & 1727.41 & 6.2214 & 0.7395 & 0.7395 & 700.75 \\
$2.5 \cdot 10^{5}$ & & 1596.84 & 48.2211 & 1237.67 & 46.8531 & 0.7204 & 1330.47 \\
$5 \cdot 10^{5}$ & & 3261.65 & 112.7294 & 2645.01 & 114.5706 & 0.7082 & 2797.26 \\
$10^{6}$ & & 6765.75 & 245.9921 & 5439.83 & 246.4489 & 0.6979 & 5672.16 \\
\hline \hline
\end{tabular}

compressibilities and are close to those of SESAME [19] and Restricted Path Integral Monte Carlo (RPIMC) [7] results. The reason for this discrepancy of the two experiments is not yet completely understood and requires more detailed study [20], including independent theoretical investigations which is the aim of this paper. It is also necessary to mention other important experimental techniques such as the convergent geometry technique [6]. The experimental point obtained by Belov et al. [6] within this technique is located between the results of laser - driven and magnetically driven flyer experiments.

An Hugoniot can be also determined theoretically from the equation of state. This enables us to compare different theoretical approaches and computer simulations with experimental results, which cover a large region in the phase diagram of hydrogen. They start at temperatures of about $20 \mathrm{~K}$ and at a density of $\rho_{0}=0.171 \mathrm{~g} / \mathrm{cm}^{3}$, which corresponds to the liquid state, and go up to temperatures and densities where only free electrons and nuclei exist. To our knowledge, there is no theory or computer simulation which rigorously and consistently describes the complete region of the EOS achievable by experiments. For example, the linear mixing model (LM) of Ross [21] rather well predicts the behavior of the laser driven experiments; however it is a semi-empirical theory which interpolates between molecular and metallic states of hydrogen.

Further, the region of completely and partially ionized hydrogen can be described analytically using the methods of quantum statistics [16, 22, 23]. In such methods, an EOS is obtained from a fugacity expansion (ACTEX) [23] and modified fugacity expansions which 
are upgraded by means of quantum-field theoretical methods (leading to dynamical screening, self energy and lowering of the ionization energy [16, 22]). In the latter case it is useful to condense the results in form of Padé approximations [24], (from Debye to GellmanBrueckner). Of course, the EOS following from these theories cannot reproduce the Hugoniot over the full range of density and pressure. It gives only the asymptotic behavior at higher temperatures. The typical behavior of the analytical theory [24] is shown in Fig. 1]. It coincides only asymptotically with the $a b$ initio RPIMC calculations and, with decreasing temperature, deviates considerably from those results. The Hugoniot calculated within the ACTEX theory which is not shown here exhibits a similar behavior [23].

The main reason for the failure of the analytical theories is obvious. As we mentioned already, for lower temperatures, the neutral particles, i.e., $\mathrm{H}$-atoms and $\mathrm{H}_{2}-$ molecules, become increasingly important, giving rise to a strongly coupled dense gas or liquid. Under such conditions it is necessary to invoke the methods of the theory of liquids. The simplest theory for this region is the fugacity expansion of the EOS up to the second virial coefficient [25]. This theory is applicable only for low densities and cannot correctly describe the molecular dissociation which is an important process occurring during shock wave experiments. For moderate densities, the fluid variational theory (FVT), proposed by Ross et al. 26], can be applied. This theory was further developed by Juranek and Redmer [12] to many component

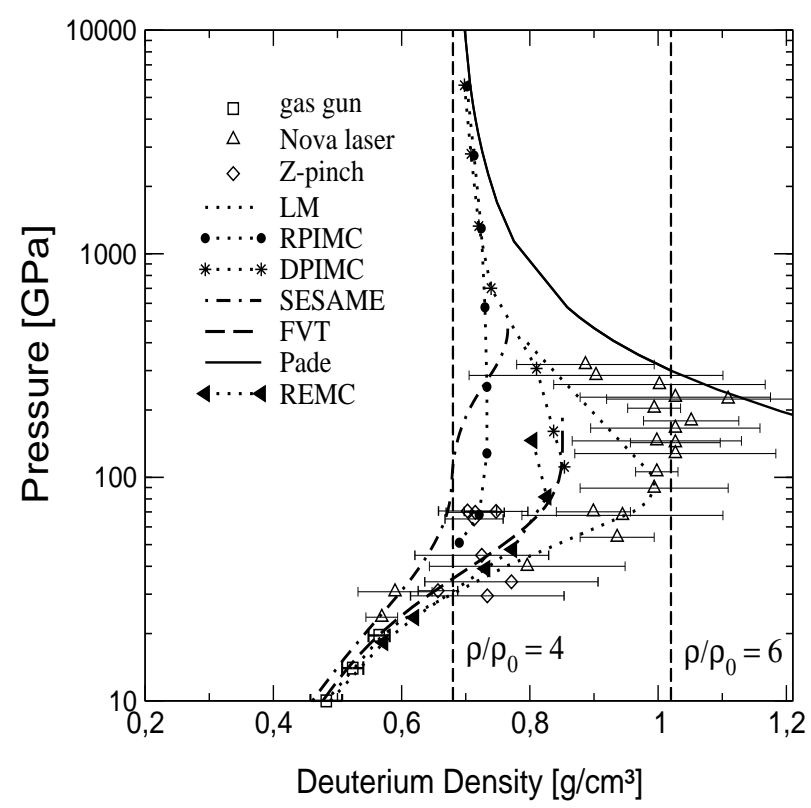

FIG. 1: Experimental and theoretical results for the deuterium Hugoniot 
systems, where molecular dissociation occurs. The effective interaction potentials [26, 27] between components used within FVT achieve good agreement with experimental gas gun data of Nellis et al. [3], Fig. 1]

A powerful tool for the investigation of the hydrogen EOS is ab initio computer simulation. Quantum molecular dynamics simulations, based on a density functional theory, are usually applied to investigate the atomic and molecular region [8, 13] but have difficulties to describe the partially ionized plasma. The wave packet molecular dynamics also covers the region of the fully ionized plasma 9] but yields unexpectedly high compressibilties. In this work we will not discuss these methods in detail and refer to the work cited.

The Path Integral Monte Carlo method is another first principle method which is well suited for the investigation of the EOS of hydrogen over a wide parameter range. Except for the problem of the Fermi statistics, it is an exact solution of the many-body quantum problem for a finite system in thermodynamic equilibrium. The treatment of the "sign problem" makes the main difference between the RPIMC method used by Ceperley and Militzer [7] and the Direct Path Integral Monte Carlo (DPIMC) method used by Filinov et al. [10, 11] and others. This problem is beyond the present paper, here we restrict ourselves to the discussion of the DPIMC method.

The idea of DPIMC is the well known: any thermodynamic property of a two-component plasma with $N_{e}$ electrons and $N_{p}$ protons at a temperature $T$ and volume $V$ is defined by the partition function $Z\left(N_{e}, N_{p}, V, T\right)$ :

$$
Z\left(N_{e}, N_{p}, V, T\right)=\frac{1}{N_{e} ! N_{p} !} \sum_{\sigma} \int_{V} d q d r \rho(q, r, \sigma ; T)
$$

where $q(r)$ comprises the coordinates of the protons (electrons), $\sigma$ stands for the spin of the electrons, and $\rho$ is the density matrix of the system. Taking into account the electron spin and the Fermi statistics (antisymmetrization), the density matrix is expressed by a path integral [28] where all electrons are represented by fermionic loops with a number of intermediate coordinates (beads). In our simulations, we used an effective quantum pair potential, which is finite at zero distance [29]. This potential was obtained by Kelbg as a result of a first-order perturbation theory. The simulation has been performed at temperatures of $10^{4} \mathrm{~K}$ and higher in a wide range of particle densities. Under these conditions the exchange effects for protons are negligible. In the present calculations, we used an improved treatment of the electron exchange, i.e., we took into account the exchange interaction of electrons from 
TABLE II: Hugoniot data calculated by REMC

\begin{tabular}{|c|c|c|c|c|c|c|c|}
\hline$T, \mathrm{~K}$ & 2000 & 4000 & 5000 & 8000 & 10000 & 13000 & 15000 \\
\hline$\rho, \mathrm{g} / \mathrm{cm}^{3}$ & 0.470 & 0.570 & 0.618 & 0.729 & 0.771 & 0.804 & 0.815 \\
\hline$P, \mathrm{GPa}$ & 9.183 & 18.690 & 23.96 & 39.35 & 47.823 & 58.71 & 65.43 \\
\hline
\end{tabular}

neighbor Monte Carlo cells, namely first from the nearest neighbor cells $\left(3^{3}-1\right)$, then from the next neighbors $\left(5^{3}-1\right)$ and so on. The calculated thermodynamic properties of hydrogen allowed us to compute the shock Hugoniot of deuterium using Eq. (3)

$$
H=E-E_{0}-\frac{1}{2}\left(p+p_{0}\right)\left(V-V_{0}\right)=0
$$

Following the work [7] we chose $p_{0}=0, \rho_{0}=0.171 \mathrm{~g} / \mathrm{cm}^{3}, E_{0}=-15.886 \mathrm{eV}$ per atom and computed the pressure $p_{i}$ and the energy $E_{i}$ at a given constant temperature $T$ (from $10^{4} \mathrm{~K}$ to $10^{6} \mathrm{~K}$ ) and three values of the volume $V_{i}=1 / \rho_{i}$ corresponding to $r_{s}=1.7,1.86$, and 2 , where $r_{s}=\bar{r} / a_{B}, \bar{r}=\left(3 / 4 \pi n_{p}\right)^{1 / 3}, n_{p}$ is the particle density, $a_{B^{-}}$Bohr radius. The results of the calculations are presented in Table 1 . Substituting the obtained values $p_{i}, E_{i}$ and $V_{i}$ into the Hugoniot we determine the volume range $V_{1}, V_{2}$ where the function $H(p, V, E)$ changes its sign. The value of the density at the Hugoniot is calculated by linear interpolation of the function $H$ between $V_{1}$ and $V_{2}$. The values of the pressure and of the total energy are shown in the Table 1 only for those density values between which the value of the density lies on the Hugoniot at a given temperature. The values of density and pressure on the Hugoniot are placed in the last two columns of Table 1. and are polotted together with selected theoretical and experimental data in Fig. 1. The lowest temperature included in this figure for the DPIMC is $15625 \mathrm{~K}$.

In order to correctly describe the quantum mechanics of the formation of molecules at temperatures lower than $10000 \mathrm{~K}$, it is necessary to take many beads. In this region, DPIMC calculations become very time consuming and the convergence is poor. The natural proposal which appears for this region is to use the asymptotic property of the path integral which, for heavy particles, goes over into the classical partition function. For such systems, the classical Monte Carlo scheme can be applied. An advanced version of the classical Monte Carlo scheme is the reaction ensemble Monte Carlo technique (REMC) [30]. This method incorporates the quantum mechanical description of bound states, while the scattering states 


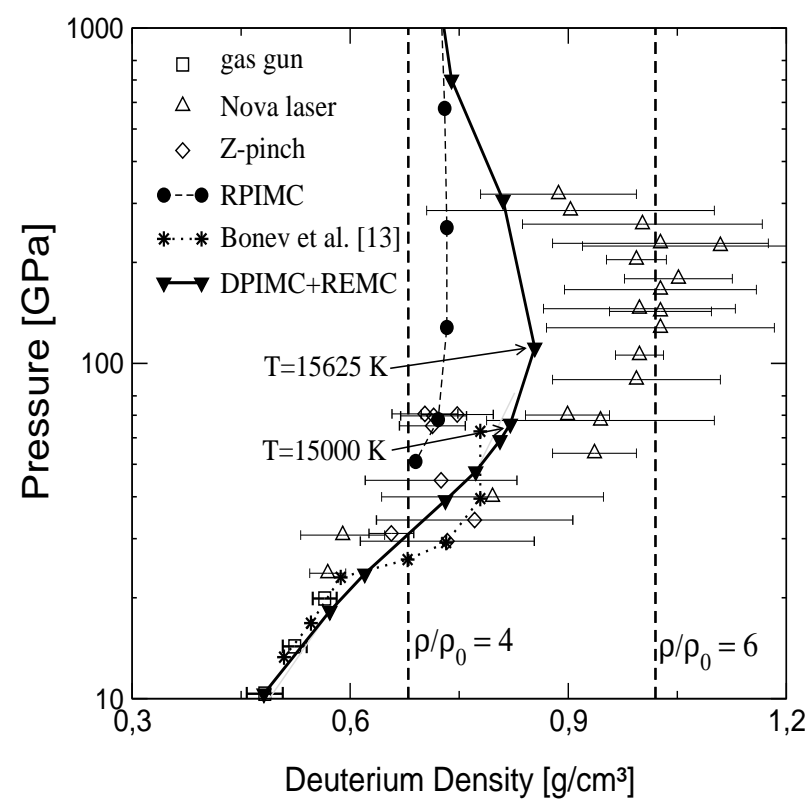

FIG. 2: Results for the combined Hugoniot

are treated classically. As was shown by Bezkrovniy et al. 14], REMC describes the low temperature region very well, and yields good agreement with the gas gun experiments by Nellis et al. [3] Fig. 1. In these simulations the energy levels for the molecular partition functions of hydrogen and deuterium are obtained by solving the Schrödinger equation with the potential calculated by Kolos and Wolniewicz [31]. On the basis of the REMC, results are obtained much easier as compared to those from molecular dynamics based on density functional theory; see Bonev et al. 13] and Fig. 2. Our REMC data are presented in Table 2 .

In order to get a unified picture combining DPIMC and REMC, we use the fact that REMC turns out to be the limiting case of DPIMC at low temperatures, where hydrogen consists only of atoms and molecules. Therefore, it is obvious to use the asymptotic results of both methods to construct an Hugoniot which can be applied in the entire range of compression. For the construction of the combined Hugoniot we carefully analyzed the region where the Hugoniots produced by the two methods can be connected to each other. As we can see from Fig. 1 the Hugoniot calculated within DPIMC ends at the point $15625 \mathrm{~K}$. At this temperature, the largest contribution to the EOS are given by molecular states. As natural continuation of the DPIMC Hugoniot, we take the point of $15000 \mathrm{~K}$ produced by REMC. We want to stress here that these two methods are completely independent and 
no interpolation procedure is used. Just two points at $15625 \mathrm{~K}$ of DPIMC and $15000 \mathrm{~K}$ of REMC are connected to each other. The final Hugoniot is plotted in Fig. 2 and shows a maximum compressibility of approximately 4.75 as compared to the initial deuterium density.

\section{Acknowledgments}

The authors greatfully acknowledge fruitful discussions with R. Redmer, Th. Bornath, and H. Juranek (Rostock). The work was supported by the Deutsche Forschungsgemeinschaft (SFB 198, BO 1366/2), the grant for talented young researchers of the Science support foundation, Rus. Fed. President Grant No. MK-1769.2003.08, the RAS program No. 17 "Parallel calculations and multiprocessor computational systems", grant of the U.S. Civilian Research and Development Foundation for Independent States of the Former Soviet Union (CRDF) No. PZ-013-02 and the Ministry of Education of Russian Federation and by a grant for CPU time at the NIC Jülich.

[1] M. D. Knudson, D. L. Hanson, J. E. Bailey, C. A. Hall, J. R. Asay and W. W. Anderson, Phys. Rev. Lett. 87, 225501-1 (2001).

[2] G. W. Collins, L. B. Da Silva, P. Celliers, D. M. Gold, M. E. Foord, R. J. Wallace, A. Ng, S. V. Weber, K. S. Budil and R. Cauble, Science 281, 1178 (1998).

[3] W. J. Nellis, A. C. Mitchell, M. van Thiel, G. J. Devine and R. J. Trainor, J. Chem. Phys., 79, 1480 (1983).

[4] L. B. Da Silva, P. Celliers, G. W. Collins, K. S. Budil, N. C. Holmes, T. W. Barbee, B. A. Hammel, J. D Kilkenny, R. J. Wallace, M. Ross, R. Cauble, A. Ng and G. Chiu, Phys. Rev. Lett. 78, 483 (1997).

[5] P. M. Celliers, G. W. Collins, L. B. Da Silva, D. M. Gold, R. Cauble, R. J. Wallace, M. E. Foord, and B. A. Hammel, Phys. Rev. Lett. 84, 5564 (2000).

[6] Belov, S. I., Boriskov, G. V., et al., Compression of solid deuterium by megabar pressures of shock waves, in Substances, Materials and Constructions under Intensive Dynamic Influences, edited by A. L. Mikhailov, VNIIEF, Sarov, 2003, pp. 100-104 
[7] B. Militzer and D. M. Ceperley, Phys. Rev. Lett., 85, 1890 (2000).

[8] L. A. Collins, S.R. Bickham, J. D. Kress, S. Mazevet, T. J. Lenosky, N. J. Troullier and W. Windl, Phys. Rev. B, 63184110 (2001).

[9] M. Knaup, P. G Reinhard and C. Toepffer, Contrib. Plasma Phys. 41, 159 (2001).

[10] V. S. Filinov, M. Bonitz and V. E. Fortov, JETP Letters, 72245 (2000).

[11] V. S. Filinov, M. Bonitz, W. Ebeling and V. E. Fortov, Plasma Phys. Cont. Fusion, 43, $743(2001)$.

[12] H. Juranek, R. Redmer and Y. Rosenfeld, J. Chem. Phys., 117, 1768 (2002).

[13] S. A. Bonev, B. Militzer and G. Galli, Phys. Rev. B, 69014101 (2004).

[14] V. Bezkrovniy, M. Schlanges, D. Kremp and W. D. Kraeft, Phys. Rev. E, accepted (2004).

[15] G. E. Norman and A. N. Starostin, Teplofizika vysokikh temperatur, 6410 (1968).

[16] W. Ebeling, W. D. Kraeft and D. Kremp, Theory of Bound States and Ionization Equilibrium in Plasmas and Solids (Akademie-Verlag, Berlin) (1976).

[17] P. Haronska, D. Kremp and M. Schlanges, Wiss. Zeitschr. Uni. Rostock, 36, 98 (1987);

[18] D. Saumon and G. Chabrier, Phys. Rev. A, 44, 5122 (1991); D. Saumon and G. Chabrier, Phys. Rev. A, 46, 2084 (1992).

[19] G. I. Kerley in Molecular-Based Study of Fluid, edited by J. M: Haile and G. A. Mansoori (American Chemical Society, Washington), 107-138 (1983).

[20] W. J. Nellis, Phys. Rev. Lett., 89, 165502 (2002).

[21] M. Ross, Phys. Rev. B, 58, 669 (1998).

[22] W. D. Kraeft, D. Kremp, W. Ebeling and G. Röpke, Quantum Statistics of Charged Particle System (Akademie-Verlag, Berlin, 1986).

[23] F. J. Rogers and D. A. Young, Phys. Rev. E, 56, 5876 (1997).

[24] W. Ebeling and W. Richert, Annalen der Physik, 39,362 (1982).

[25] M. Schlanges and D. Kremp, Annalen der Physik, 39, 69 (1982).

[26] M. Ross, F. H. Ree and D. A. Young, J. Chem. Phys. 79, 1487 (1983).

[27] F. H. Ree, in Shock Waves in Condensed Matter, editors S. C. Schmidt, N. C. Holmes (Elsevier Science Publishers), 125 (1988).

[28] Zamalin, V. M., Norman, G. E., and Filinov, V. S., The Monte-Carlo Method in Statistical Thermodynamics, Nauka, Moscow, 1977 
[29] Filinov, V. S., Bonitz, M., Levashov, P. R., Fortov, V. E., Ebeling, W., Schlanges, M., and Koch, S. W., J. Phys. A.: Math. Gen., 36, 6069-6076 (2003).

[30] W. R. Smith and B. Triska, J. Chem. Phys., 100, 3019 (1994); J. K. Johnson, A. Panagiotopoulos and K. E. M. Gubbins, Mol. Phys., 81, 717 (1994).

[31] W. Kolos and L. Wolniewicz, J. Chem. Phys., 43, 2429 (1965). 